Scientific Paper

\title{
Attenuation parameters of polyvinyl alcohol-tungsten oxide composites at the photon energies 5.895, 6.490, 59.54 and $662 \mathrm{keV}$
}

\author{
Muthamma M V ${ }^{1}$, Bubbly S GudenNAVAR ${ }^{1, a}$, Shivappa B GUdENNAVAR ${ }^{1}$ \\ ${ }^{l}$ Department of Physics and Electronics, CHRIST (Deemed to be University), Bangalore Central Campus, \\ Bengaluru-560029, Karnataka, India \\ ${ }^{a}$ E-mail address: bubbly.sg@christuniversity.in
}

(received 5 April 2019; revised 18 November 2019 and 8 January 2020; accepted 11 March 2020)

\begin{abstract}
The growing demand for lightweight, non-toxic and effective $\mathrm{X}$ - and $\gamma$-ray shielding materials in various fields has led to the exploration of various polymer composites for shielding applications. In this study, tungsten filled polyvinyl alcohol (PVA) composites of varying $\mathrm{WO}_{3}$ concentrations $(0-50 \mathrm{wt} \%)$ were prepared by solution cast technique. The structural, morphological, and thermal properties of the prepared composite films were studied using X-ray diffraction technique (XRD), Scanning electron microscopy (SEM) and Thermogravimetric analysis (TGA). The AC conductivity studies showed the low conductivity property of the composites. The X-ray (5.895 and $6.490 \mathrm{keV})$ and $\gamma$-ray (59.54 and $662 \mathrm{keV}$ ) attenuation studies performed using $\mathrm{CdTe}$ and $\mathrm{NaI}(\mathrm{Tl})$ detector spectrometers revealed a noticeable increase in shielding efficiency with increase in filler wt $\%$. The effective atomic number $\left(\mathrm{Z}_{\mathrm{eff}}\right)$ calculated by the direct method agreed with the values obtained using Auto- $Z_{\mathrm{eff}}$ software. The $\%$ heaviness showed that tungsten filled polyvinyl alcohol composites are lighter than traditional shielding materials.
\end{abstract}

Key words: radiation shielding; tungsten filled polyvinyl alcohol composite; mass attenuation coefficient.

\section{Introduction}

With the advancement in science and technology, the increased usage of ionizing radiation in many areas has raised the global issues of radiation exposure and risks. In order to keep the radiation exposure to a minimum and to ensure that they do not cause undue risk to human beings, adequate shielding has to be employed. Protection of scientific instruments on-board satellites against harsh radiations in space environments also requires efficient and lightweight shields. High-density lead and concretes have been traditionally used as radiation shields in radiation facilities. While health concerns discourage the use of toxic lead, the usage of concrete has space constraints. Different glass systems incorporating heavy metals have been investigated for radiation shielding [1-5] as they have the advantage of less toxicity and being transparent to visible light. Significant research efforts towards designing lightweight, flexible, and cost-effective radiation shields have focused on polymer composites for radiation shielding applications [6-9]. Toxicity of lead being a major concern, lead-free polymer composites reinforced with high $\mathrm{Z}$ fillers have gained considerable attention from researchers in recent years [10-16]. There are many reports on polymer composites of bismuth and tungsten being investigated for radiation shielding [15,17-19].
The present work has focused on quantitative analysis of shielding efficiency of flexible, lightweight, polyvinyl alcohol (PVA) composites with varying wt\% (0-50) of tungsten. PVA being water-soluble polymer has varied applications in industrial markets such as textiles, coatings, paper, ceramics, and wood. The non-toxic, ductile but strong and flexible nature with effective film-forming properties makes it a unique polymer with few matching substitutes. In the search for ecofriendly polymers, PVA has become economical due to its biodegradability under certain microbial conditions. The choice of tungsten as filler was made due to its high density, nonreactivity during chemical functionalization and radiation shielding capabilities. The synthesized composites have been characterized by XRD and SEM for their structural properties. They have also been studied for their thermal, optical, and electrical behavior, which reveal their suitability as radiation shields. The mass attenuation coefficients of the composites have been determined experimentally at the incident $\mathrm{X}$ - and $\gamma$ ray energies of 5.89, 6.49, 59.54, and $662 \mathrm{keV}$ and compared with theoretical values computed using WinXCom [20]. The effective atomic number and \% heaviness of the composites have also been determined. 


\section{Materials and methods}

\section{Materials and preparation}

PVA- $\mathrm{WO}_{3}$ composites have been prepared by dispersing tungsten trioxide (MW $\sim 231.837 \mathrm{~g} \mathrm{~mol}^{-1}$ ) in methanol and adding into an aqueous solution of polyvinyl alcohol (MW 1, 25, $000 \mathrm{~g} \mathrm{~mol}^{-1}$ ) (S D Fine Chem Ltd). The solutions prepared with different wt $\%(5,10,20,30,40,50)$ of $\mathrm{WO}_{3}$ were stirred at a constant speed using a magnetic stirrer until the solutions turned viscous to ensure uniform dispersion of $\mathrm{WO}_{3}$ into PVA matrix. The viscous solution was cast to circular glass petridish (5-inch diameter) and allowed to dry at room temperature. These films were then peeled off and cut to desired dimensions for further studies.

\section{Structural and thermal characterization}

The X-ray diffraction data of the composite films were obtained in $2 \theta=0-120^{\circ}$ scan mode with $\mathrm{Cu}$ source $(\lambda=1.5406$ $\AA$ ) using Bruker AXS D8 Advance X-ray diffractometer. Highresolution SEM images were obtained from JEOL JSM6390LV to know the distribution of filler in the PVA matrix. TGA studies of the composites were carried out using Perkin Elmer Diamond TG/DTA (ambient temperature to $1200^{\circ} \mathrm{C}$ ) in the temperature range $50-700{ }^{\circ} \mathrm{C}$. Optical absorption studies on the composites were carried out using Varian Cary 5000 UVvis spectrophotometer. The AC conductivity measurements were conducted using LCR meter (LCR-8000G series GWInstek) in the frequency range $1 \mathrm{~Hz}-32 \mathrm{MHz}$ at room temperature.

\section{$\mathrm{X}$ - and $\boldsymbol{\gamma}$-ray attenuation studies}

$\mathrm{X}$-rays interact with matter mainly via photoelectric effect, Compton scattering and Rayleigh scattering. The two interaction processes dominant in the diagnostic energy range of X-rays are photoelectric effect and Compton scattering. Similarly, $\gamma$-rays can interact with a medium through photoelectric effect, Compton scattering, and pair production processes. The interaction cross-section of these processes mainly depends on the energy of the incident radiation $\left(\mathrm{E}_{\gamma}\right)$ and atomic number $(\mathrm{Z})$ of the medium. The attenuation of $\mathrm{X}-/ \gamma$-rays in a medium is governed by the exponential law (Equation 1):

$\mathrm{I}=\mathrm{I}_{0} \mathrm{e}^{-(\mu / \rho) \mathrm{t}}$

Eq. 1

where, $\mathrm{I}_{0}$ and $\mathrm{I}$ are the intensities of the incident and transmitted $\mathrm{X}-/ \gamma$-rays, $\mathrm{t}$ is the mass thickness $\left(\mathrm{g} \mathrm{cm}^{-2}\right)$ and $\mu / \rho$ is the mass attenuation coefficient $\left(\mathrm{cm}^{2} \mathrm{~g}^{-1}\right)$ of the medium for $\mathrm{X}-/ \gamma$-rays of a given energy.

$\mathrm{X}$ - and $\gamma$-ray attenuation behavior of the polymer composites were studied using ${ }^{55} \mathrm{Fe}(5.895 \mathrm{keV}$ and $6.490 \mathrm{keV}),{ }^{241} \mathrm{Am}$ $(59.54 \mathrm{keV})$ and ${ }^{137} \mathrm{Cs}(662 \mathrm{keV})$ point radioactive sources, and Amptek XR-100T-CdTe and $2 " \times 2 " \quad \mathrm{NaI}(\mathrm{Tl})$ detector spectrometers adopting narrow beam geometry [21]. In this arrangement, lead collimators of suitable diameter and thickness were used to collimate the $\mathrm{X}$ - and $\gamma$-ray beam from the radioactive sources. The samples were placed midway between the source and the detector. The radioactive $\mathrm{X}$ - and $\gamma$ ray sources used in the present investigation were procured from the Board of Radiation and Isotope Technology, Bombay, India, as standard $\mathrm{X}$ - and $\gamma$-ray sources. A CdTe/NaI(Tl) detector coupled to an active filter amplifier and a $1 \mathrm{k}$ multichannel analyzer was used to measure the incident and transmitted X-/ $\gamma$-ray intensities. These detector spectrometers were calibrated using $\mathrm{X}$ - and $\gamma$-rays of different energies. The linearity and stability of the spectrometers were checked intermittently throughout the experiments.

In order to standardize the methodology with CdTe X-ray detector spectrometer, an experiment was conducted to determine the mass attenuation coefficient $(\mu / \rho)$ for the X-ray $\left(\mathrm{K}_{\alpha}\right.$ and $\mathrm{K}_{\beta}$ ) of energies 5.895 and $6.490 \mathrm{keV}$ from ${ }^{55} \mathrm{Fe}$ source and $59.54 \mathrm{keV} \gamma$-ray from ${ }^{241} \mathrm{Am}$ source taking aluminum targets of varying thickness as reference. Similarly, the methodology with $\mathrm{NaI}(\mathrm{Tl})$ detector spectrometer was standardized by conducting experiment to determine the $\mu / \rho$ values for the $662 \mathrm{keV} \gamma$-ray from ${ }^{137} \mathrm{Cs}$ source taking aluminum as reference. $\mu / \rho$ values at various energies of $X-/ \gamma$ rays were determined from the plots of $\ln (\mathrm{I})$ versus mass thickness $(\mathrm{t})$. The $\mu / \rho$ values were determined in three trials and the weighted mean value of $\mu / \rho$ was compared with standard theoretical $\mu / \rho$ value computed using WinXCom [20]. The $\mu / \rho$ values for aluminum at selected energies agreed well with the standard theoretical values computed from WinXCom [20] and others' experimental values available in the literature. $X-/ \gamma$-ray attenuation experiments on the composites were performed varying the mass thickness of the polymer composites and measuring the transmitted intensities (I).

An effective atomic number $\left(\mathrm{Z}_{\mathrm{eff}}\right)$ of the polymer composite is an important parameter to be considered in designing radiation shields and computing an absorbed dose of radiation. $\mathrm{Z}_{\text {eff }}$ values of the composites were calculated using experimental $\mu / \rho$ values in the following semi-empirical relation (Equation 2) based on mixture rule:

$Z_{e f f}=\frac{\sigma_{a}}{\sigma_{e l}}$

Eq. 2

where $\sigma_{a}=\frac{1}{N_{A}} \frac{\frac{\mu}{\rho}}{\sum_{i}\left(\frac{w_{i}}{A_{i}}\right)}$ and $\sigma_{e l}=\frac{1}{N_{A}} \sum_{i} \frac{f_{i} A_{i}}{Z_{i}}\left(\frac{\mu}{\rho}\right)_{i}$ are the average cross-section per atom (b/atom) and average cross-section per electron (b/electron) respectively, $N_{A}$ is the Avogadro's constant, $w_{i}=\frac{n_{i} A_{i}}{\sum_{i} n_{i} A_{i}}$ is weight fraction, $n_{i}$ the number of atoms, $A_{i}$ the atomic mass, and $Z_{i}$ the atomic number of the $\mathrm{i}^{\text {th }}$ constituent element. $\left(\frac{\mu}{\rho}\right)_{i}$ is the mass attenuation coefficient of the ith constituent element obtained using WinXCom program and $f_{i}=\frac{n_{i}}{\sum_{i} n_{i}}$ is the fractional abundance of $\mathrm{i}^{\text {th }}$ element with respect to the total number of atoms $\left(\sum_{i} n_{i}=n\right)$ in the molecular formula such that $\sum_{i} f_{i}=1$. The tool Auto- $\mathrm{Z}_{\mathrm{eff}}$ in 
Visual Basic was also used for determining $\mathrm{Z}_{\mathrm{eff}}$ of the composites for the photon energies ranging from $1 \mathrm{keV}-1$ $\mathrm{GeV}[22]$.

\section{Density and \% heaviness}

Densities of the composite films have been determined by measuring sample thickness, area, and mass. Sample thickness has been determined by placing the film between two uniform glass plates to avoid rupture of the film from direct measurement using a digital micrometer. The dimension $(2 \mathrm{~cm}$ $\times 2 \mathrm{~cm}$ ) of the cut sample was determined using a traveling microscope. The mass of each cut sample has been carefully measured using a high precision digital balance. Measured densities $(\rho=$ mass/(area $\times$ thickness $)$ ) of the composites were compared with theoretical densities calculated using Equation 3:

$\rho=\frac{100}{\frac{m}{\rho_{\mathrm{m}}}+\frac{\mathrm{f}}{\rho_{\mathrm{f}}}}$

Eq. 3

where, $m$ and $f$ are the wt $\%$ and $\rho_{\mathrm{m}}$ and $\rho_{\mathrm{f}}$ are the densities of the polymer matrix and filler respectively.

$\%$ heaviness of the polymer composites was determined using Equation 4 assuming lead to be the standard and normalized to $100 \%$ :

$\%$ heaviness $=\frac{\text { Density of the composite }}{\text { Density of lead }} \times 100$

Eq. 4

\section{Results and discussion}

\section{XRD analysis}

The X-ray diffractograms (Figure 1) revealed a broad intense peak at $2 \theta=19^{\circ}$ and a shoulder peak at $22^{\circ}$ for neat PVA which correspond to crystalline orthorhombic lattice signifying semi-crystalline nature $[23,24]$. The intensity of this peak has been observed to decrease in the 5-20 wt \% tungsten filled PVA composites. The diffraction peaks observed at $2 \theta=22.7^{\circ}$, $23.1^{\circ}, 24.0^{\circ}, 26.6^{\circ}, 28.6^{\circ}, 29^{\circ}, 32.9^{\circ}, 33.4^{\circ}, 34.8^{\circ}, 41.3^{\circ}, 42^{\circ}$, $47.7^{\circ}, 49.29^{\circ}, 49.9^{\circ}, 52.7^{\circ}, 53.2^{\circ}, 54.2^{\circ}, 55.48^{\circ}, 59.28^{\circ}$, and $61.6^{\circ}$ in 5-50 wt\% composites showed an increase in the intensity with a concentration of $\mathrm{WO}_{3}$ and were in good agreement with JCPDS file numbers 05-0363 and 33-1387 of tungsten trioxide [25]. The average crystallite size of the composites calculated from Scherrer equation varied from 5-29 nm.

\section{SEM analysis}

SEM image in Figure 2a shows the surface morphology of neat PVA film without any pores. Figures $\mathbf{2 b}$ and $\mathbf{2 c}$ show the surface morphology of composite films with an increased concentration of $\mathrm{WO}_{3}$.

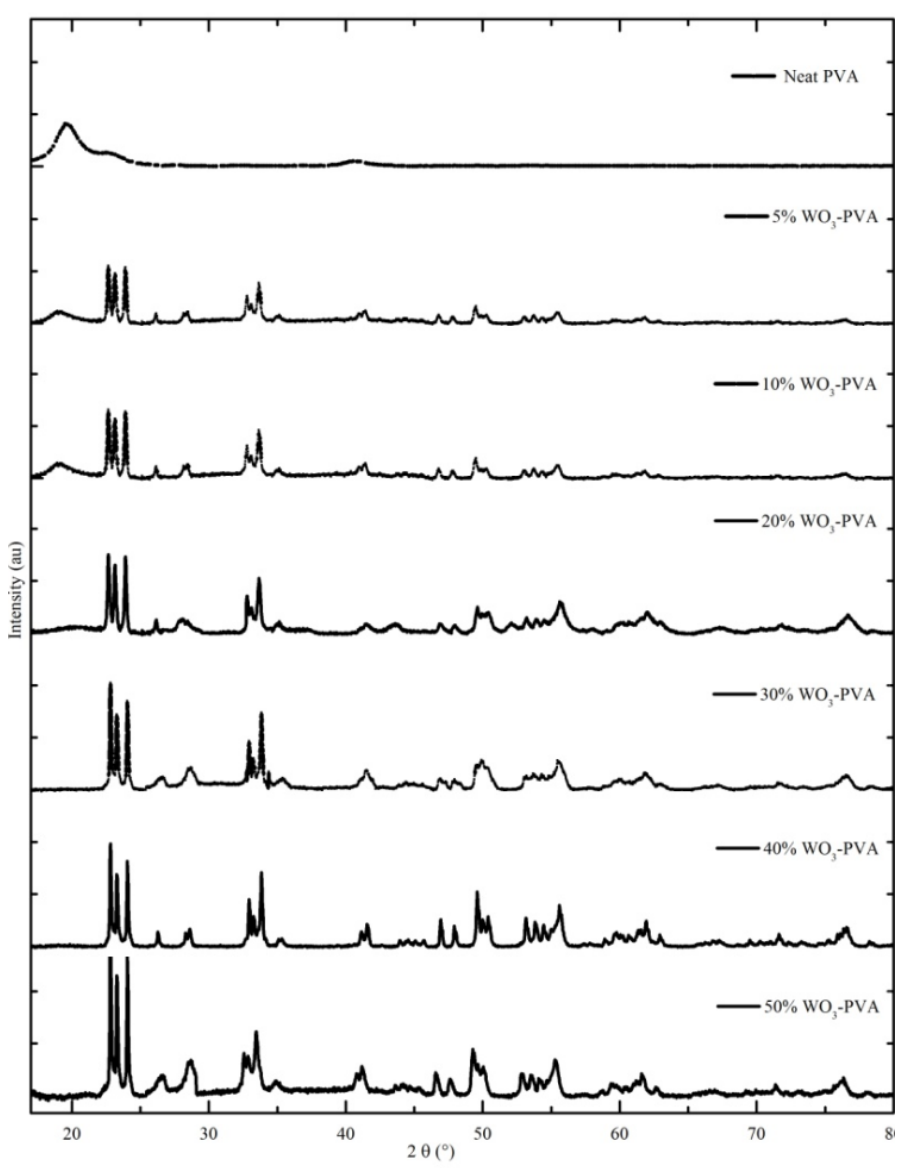

Figure 1. XRD of PVA-WO $\mathrm{WO}_{3}$ composites
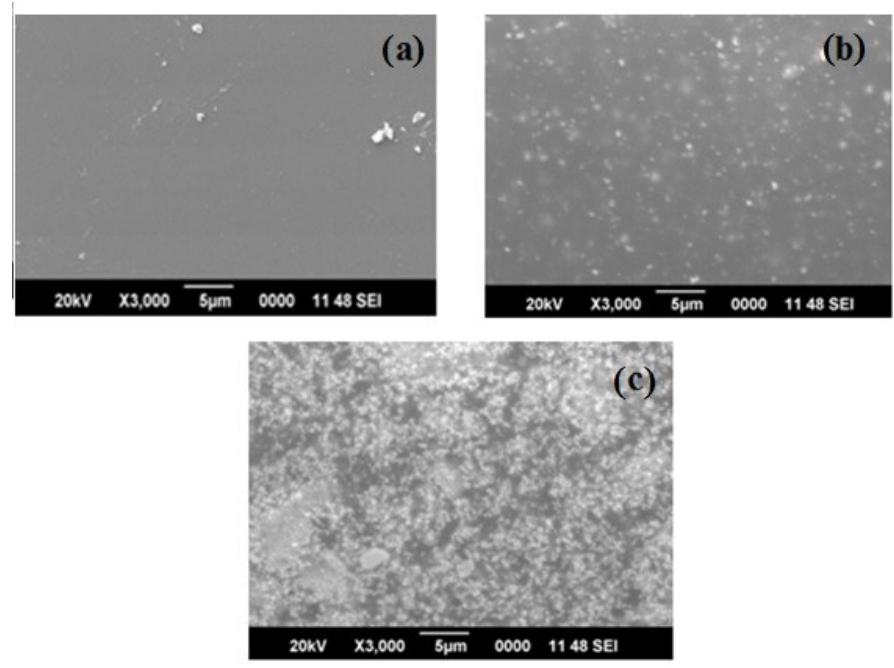

Figure 2. (a) SEM images of Neat PVA, (b) $20 \% \mathrm{WO}_{3}$ filled PVA and (c) $50 \% \mathrm{WO}_{3}$ filled PVA composites 


\section{AC conductivity measurements}

AC conductivity of the composites measured at room temperature decreased with an increase in filler concentration (Figure 3). However, the composites showed a slight increase in conductivity with an increase in frequency due to the mobility of charge carriers [26].

\section{UV-vis spectroscopic studies}

UV-vis absorption spectra for the composites are shown in Figure 4a. Neat PVA exhibited an absorption peak at $284 \mathrm{~nm}$ and a shoulder peak at $330 \mathrm{~nm}$ which may be assigned to $\pi \rightarrow \pi^{*}$ electronic transition [27]. The absorbance of PVAWO3 composites is maximum around $310 \mathrm{~nm}$, which could possibly be the band edge of maximum absorption in composites. Further, a sharp decrease in absorbance of composites was observed from 360-470 nm which could be due to a lower number of states for absorption to occur.
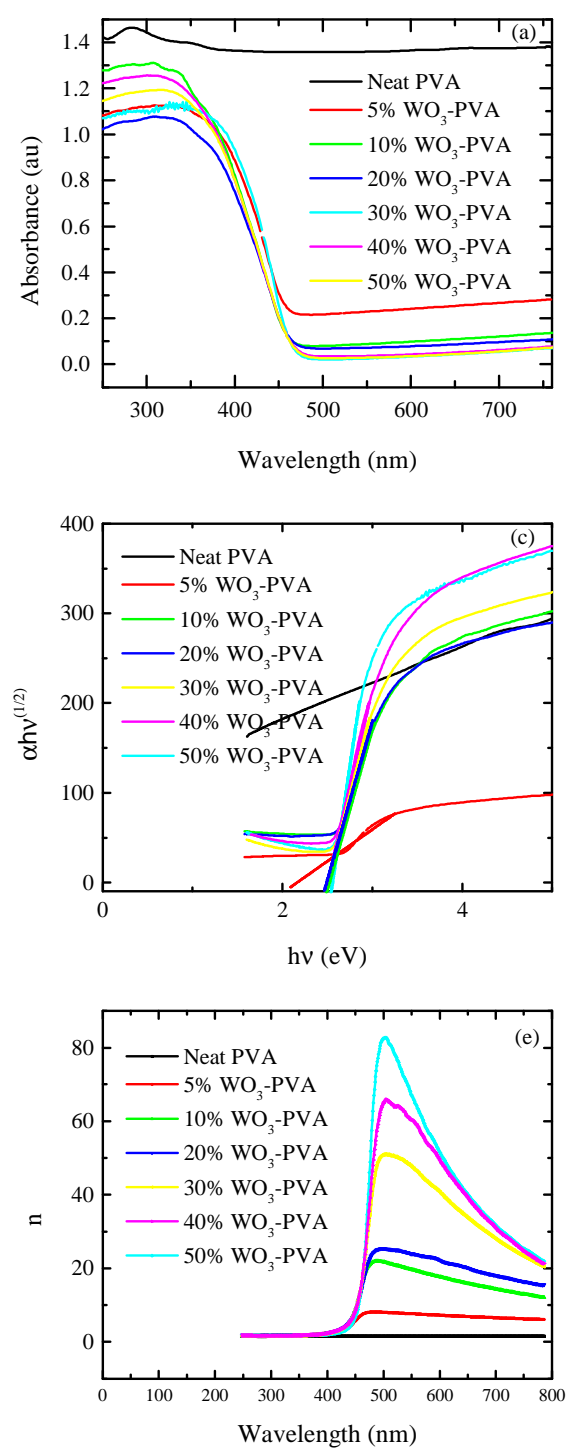
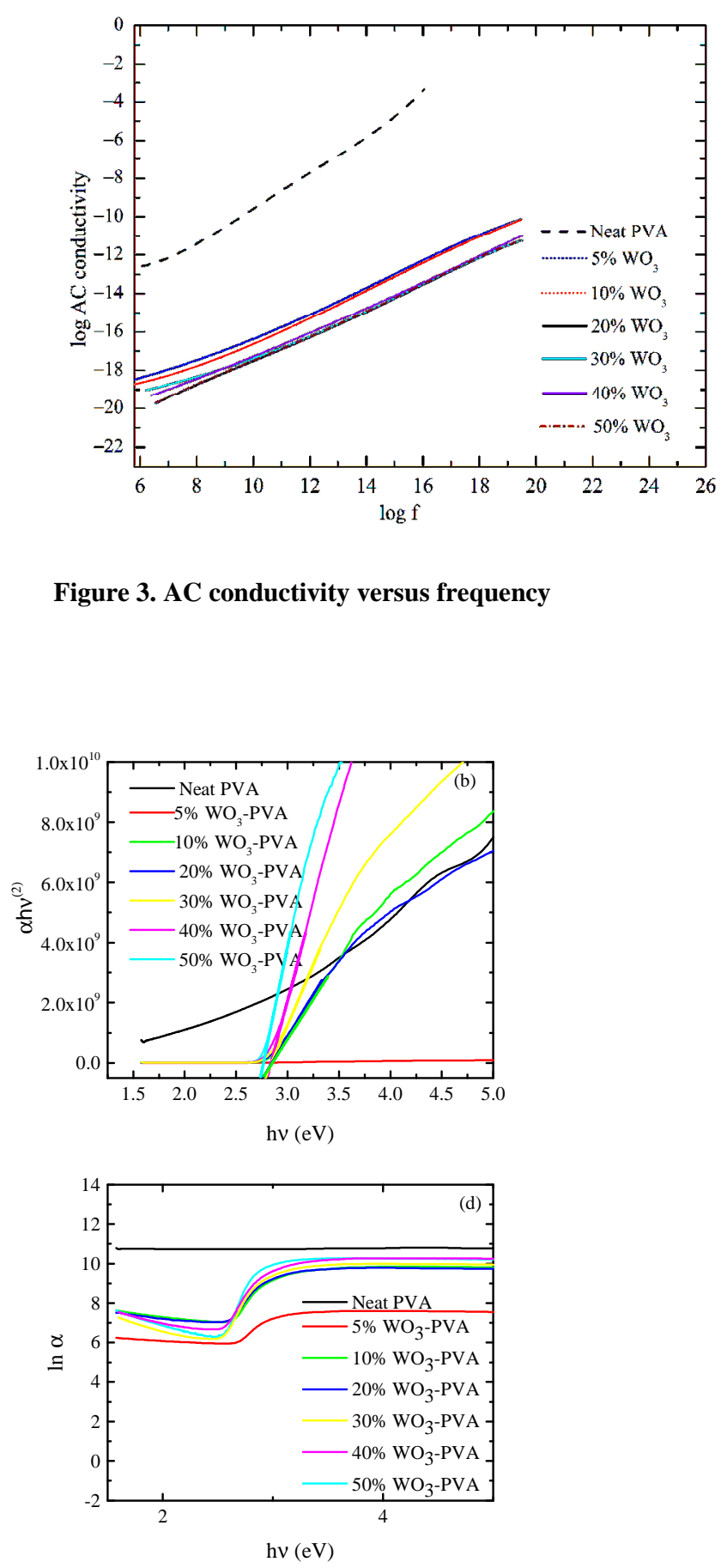

Figure 3. AC conductivity versus frequency

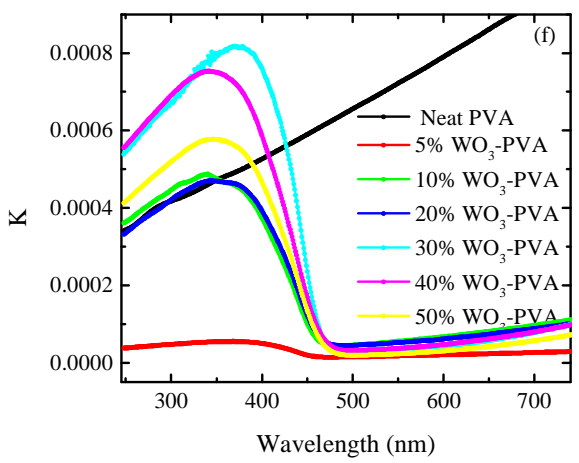

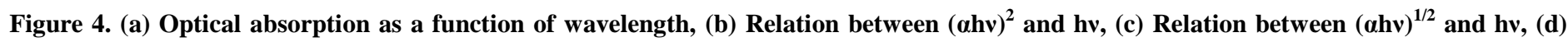
Relation between $\ln (\alpha)$ and $h v$, (e) Refractive index as a function of wavelength, (f) Extinction coefficient as a function of wavelength. 
The optical band gap energies $\left(\mathrm{E}_{\mathrm{g}}^{\mathrm{opt}}\right.$ ) for direct allowed transitions were obtained by applying linear fit to the absorption edge of $(\alpha h v)^{2}$ and $(\alpha h v)^{1 / 2}$ versus photon energy plots (Figures $\mathbf{4 b}$ and $\mathbf{4 c}$ ). The band gap energies obtained were $5.9 \mathrm{eV}$ and $\sim 2.7 \mathrm{eV}$ for neat PVA and its composites respectively. Despite the decrease in the optical energy band gap with an increase in filler concentration, the composites still showed a tendency of aligning towards insulators. The graph of the natural logarithm of absorption coefficient ( $\ln \alpha$ ) versus photon energy (hv) (Figure 4d) showed an exponential dependence indicating that the sample obeys Urbach's formula [28]. The variation of refractive index with an increase in wavelength due to interaction between the incident photons and electrons of the composite films is shown in Figure 4e. The refractive index of the composites is independent of wavelength in the UV region and shows a sharp increase in the visible region (400-500 $\mathrm{nm}$ ) revealing bulk property of the composite. Further increase in wavelength showed a decrease in the refractive index. A sudden increase in the refractive index in the visible region could be a train of percolation threshold phenomenon [29]. Fractional energy loss due to scattering and absorption known by the parameter extinction coefficient $(\mathrm{K})$ decreased with filler concentration and wavelength (Figure 4f). Further decrease in the extinction coefficient may be attributed to a decrease in scattering of photons [30].

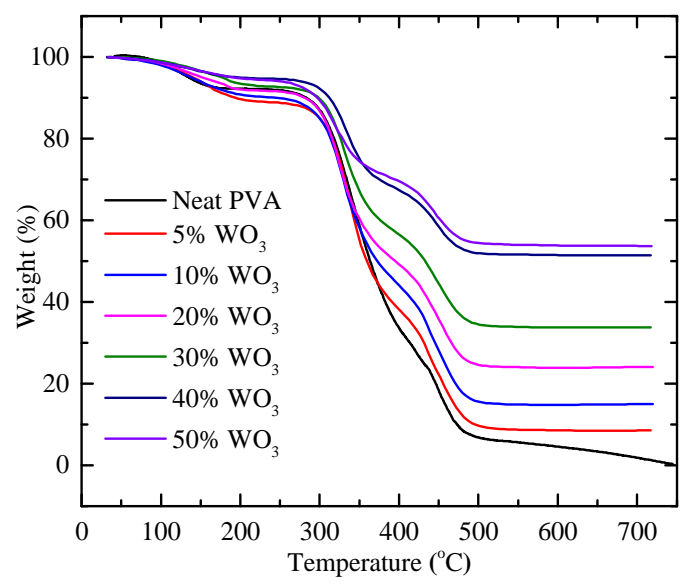

Figure 5. Variation of weight (\%) of the PVA and the composites of different $\mathrm{wt} \% \mathrm{WO}_{3}$ with temperature.

\section{Thermogravimetric analysis}

Thermogravimetric curves of composites obtained from room temperature to $700^{\circ} \mathrm{C}$ in nitrogen atmosphere are shown in Figures 5 and 6. The first weight loss of $14.7 \%$ in neat PVA and $10.6 \%-5 \%$ in composites observed around $150^{\circ} \mathrm{C}$ are attributed to the evaporation of occluded moisture. Further, due to the decomposition of the PVA component in the matrix the second sharp weight loss of $60.52 \%$ at $340^{\circ} \mathrm{C}$ for neat PVA, and a decrease in weight loss from 50.44-23.24\% around $331^{\circ} \mathrm{C}$ were observed. The third weight loss of $28 \%$ around $448^{\circ} \mathrm{C}$ was observed in neat PVA due to carbonation. The composites too experienced a weight loss of $20-16 \%$ around the same temperature due to the release of $\mathrm{CO}_{2}$. The PVA- $\mathrm{WO}_{3}$ composites showed a decrease in weight loss with an increase in filler concentration at all three stages.

\section{$\mathrm{X}$ - and $\boldsymbol{\gamma}$-ray attenuation studies}

$\mathrm{PVA}^{-} \mathrm{WO}_{3}$ composites of varying mass thickness (0.025 $0.040 \mathrm{~g} \mathrm{~cm}^{-2}$ ) were used in the attenuation experiments to determine $\mu / \rho$ values at X-ray energies 5.895 and $6.490 \mathrm{keV}$ from ${ }^{55} \mathrm{Fe}$. $\mu / \rho$ values of the composites were found to increase with filler concentration from $17-127 \mathrm{~cm}^{2} \mathrm{~g}^{-1}$ and $13-99 \mathrm{~cm}^{2} \mathrm{~g}^{-1}$ for 5.895 and $6.490 \mathrm{keV}$, respectively (Table 1).

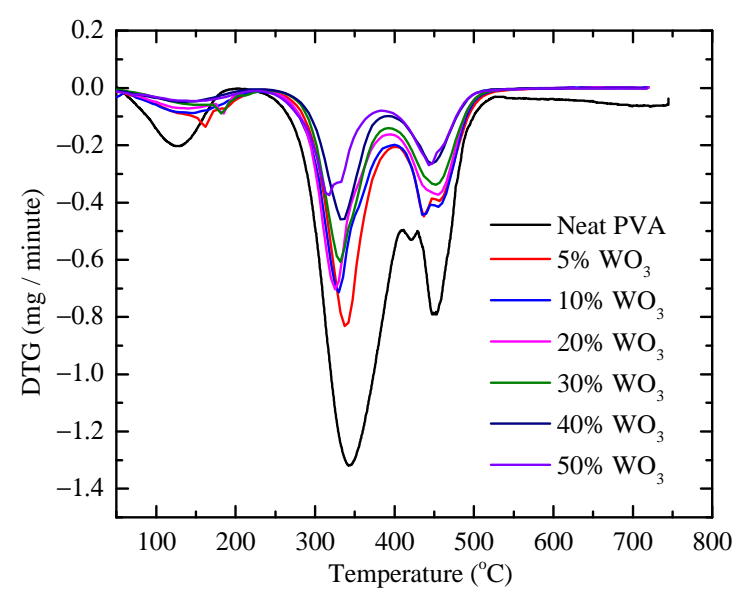

Figure 6. Derivative weight thermogram of PVA and the composite films with different $\mathrm{wt} \%$ of $\mathrm{WO}_{3}$

Table 1. The mass attenuation coefficient of $\mathrm{WO}_{3}$ filled PVA composites at various energies

\begin{tabular}{|c|c|c|c|c|c|c|c|c|}
\hline \multirow{2}{*}{$\begin{array}{c}\text { Energy } \\
(\mathrm{keV})\end{array}$} & \multirow{2}{*}{$\begin{array}{c}\text { Filler } \\
(w t \%)\end{array}$} & \multicolumn{7}{|c|}{ Mass attenuation coefficient, $\mu / \rho\left(\mathrm{cm}^{2} \mathrm{~g}^{-1}\right)$} \\
\hline & & Neat PVA & $5 \%$ & $10 \%$ & $20 \%$ & $30 \%$ & $40 \%$ & $\mathbf{5 0 \%}$ \\
\hline \multirow{2}{*}{5.895} & $E$ & $17.46 \pm 0.03$ & $30.67 \pm 0.08$ & $45.09 \pm 0.07$ & $73.30 \pm 0.62$ & $99 \pm 1$ & $127.60 \pm 0.53$ & -- \\
\hline & $\mathrm{C}$ & 17.00 & 31.00 & 45.10 & 73.10 & 101.00 & 129.00 & -- \\
\hline \multirow{2}{*}{6.490} & $E$ & $13.10 \pm 0.05$ & $23.62 \pm 0.10$ & $34.96 \pm 0.11$ & $57.14 \pm 0.40$ & $77.11 \pm 0.80$ & $99.68 \pm 0.46$ & $\begin{array}{l}- \\
-\end{array}$ \\
\hline & $\mathrm{C}$ & 12.70 & 23.70 & 34.70 & 56.80 & 78.80 & 101.00 & -- \\
\hline \multirow{2}{*}{59.54} & $E$ & -- & -- & -- & $0.75 \pm 0.01$ & $1.03 \pm 0.01$ & $1.33 \pm 0.02$ & $1.57 \pm 0.03$ \\
\hline & $\mathrm{C}$ & -- & -- & -- & 0.77 & 1.05 & 1.33 & 1.62 \\
\hline \multirow{2}{*}{662} & $\mathrm{E}$ & -- & -- & -- & -- & $0.086 \pm 0.001$ & $0.087 \pm 0.001$ & $0.088 \pm 0.001$ \\
\hline & $\mathrm{C}$ & -- & -- & -- & -- & 0.087 & 0.088 & 0.089 \\
\hline
\end{tabular}


The experiment was also performed for $\gamma$-ray energy of 59.54 $\mathrm{keV}$ of ${ }^{241} \mathrm{Am}$ source with a mass thickness of the composites varying from $0.280-0.701 \mathrm{~g} \mathrm{~cm}^{-2} . \mu / \rho$ values obtained varied from $0.65-1.57 \mathrm{~cm}^{2} \mathrm{~g}^{-1}$ with an increase in filler concentration

\section{(Table 1).}

Similarly, $\gamma$-ray attenuation studies were performed for 662 $\mathrm{keV}$ of ${ }^{137} \mathrm{Cs}$ source using $\mathrm{NaI}(\mathrm{Tl})$ detector spectrometer for varying mass thickness $\left(0.790-2.639 \mathrm{~g} \mathrm{~cm}^{-2}\right)$ and the results showed an increase in mass attenuation coefficients $(0.086$ -
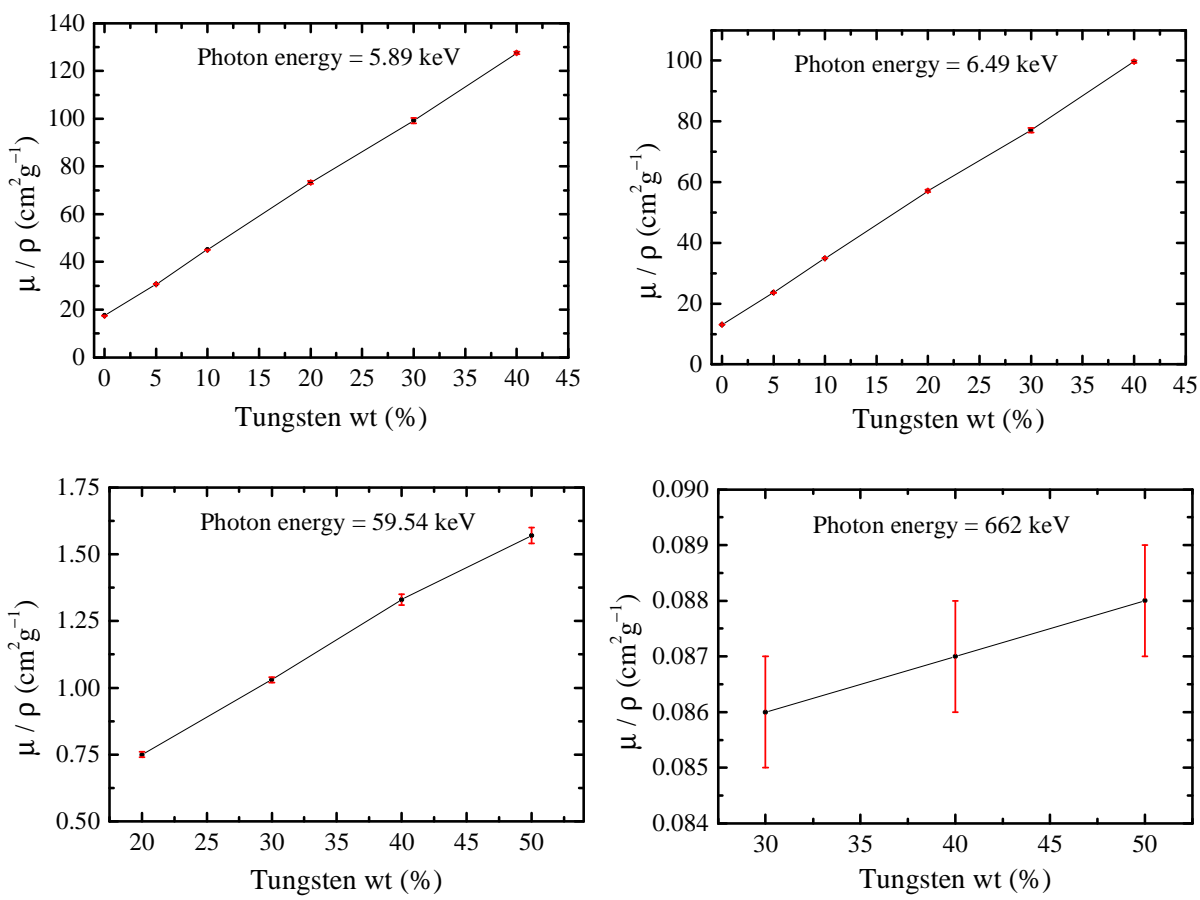

Figure 7. Variation of $\mu / \rho$ values with tungsten filler wt \% at photon energy $5.895 \mathrm{keV}, 6.49 \mathrm{keV}, 59.54 \mathrm{keV}$ and $662 \mathrm{keV}$
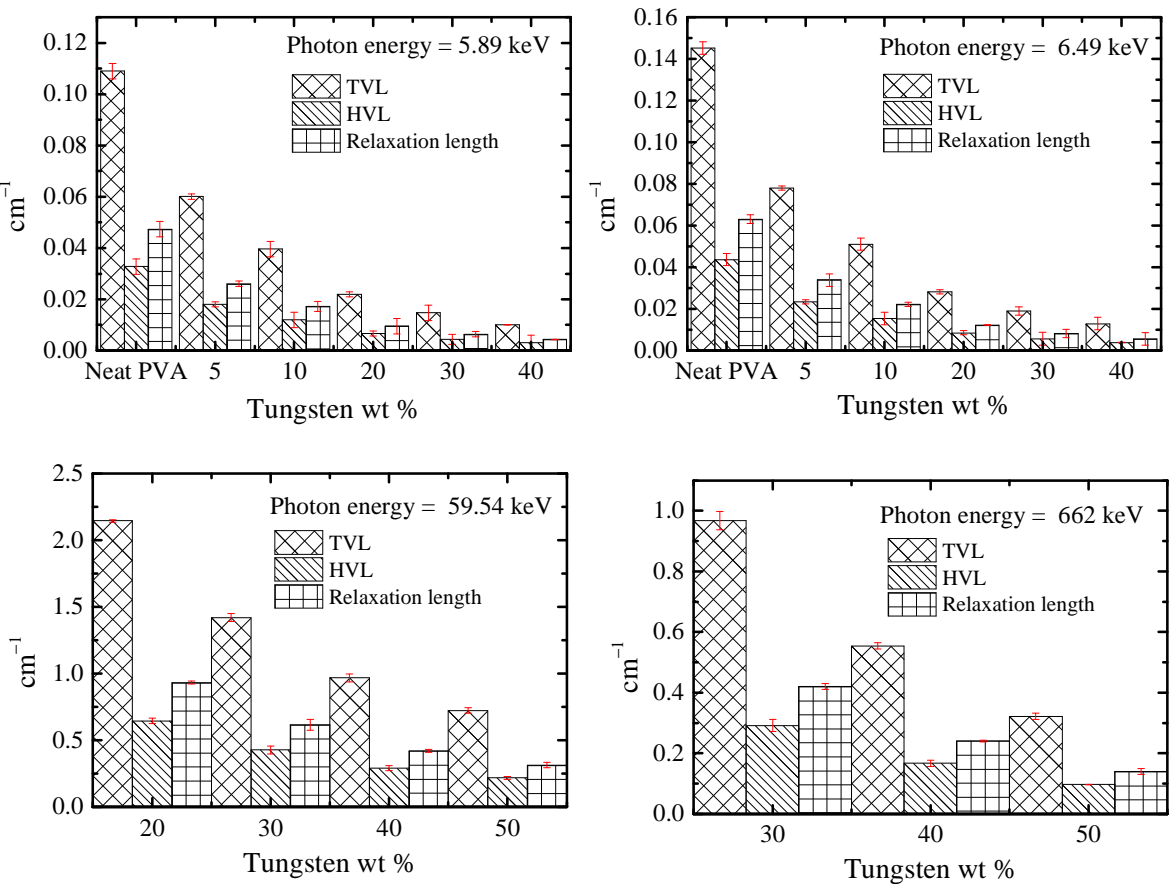

Figure 8. Half Value Layer (HVL), Tenth Value Layer (TVL) and relaxation length $(\lambda)$ with wt \% at photon energy $5.895 \mathrm{keV}, 6.49 \mathrm{keV}$, $59.54 \mathrm{keV}$ and $662 \mathrm{keV}$ 


\section{Effective atomic number $\left(Z_{\text {eff }}\right)$}

The $\mathrm{Z}_{\text {eff }}$ obtained from the ratio of atomic to electronic crosssections at 5.895, 6.490, 59.54 and $662 \mathrm{keV}$ using Equation 2 were compared with $Z_{\text {eff }}$ obtained from theoretical $\mu / \rho$ values from WinXCom (Table 2 and $\mathbf{3}$ ). The $Z_{\text {eff }}$ values were found to increase with filler concentration for all the estimated energies. The $\mathrm{Z}_{\text {eff }}$ for composites measured at 59.54 and $662 \mathrm{keV}$ were compared with the values computed using Auto- $Z_{\text {eff }}$ computer program and are reported in Tables 3 and $\mathbf{4}$ respectively. Auto$Z_{\text {eff }}$ computer program is not suitable for evaluating $Z_{\text {eff }}$ at lower photon energies such as 5.89 and $6.49 \mathrm{keV}$. The program shows notable disagreement in Zeff values for a medium of higher atomic number which was observed with PVA-WO3 composites of mass thickness $0.280-0.701 \mathrm{~g} \mathrm{~cm}^{-2}$ studied for $59.54 \mathrm{keV}$.

\section{Density and \% heaviness of the composites}

Considering lead to be $100 \%$ heavier than other shielding materials, steel is at $69.47 \%$, barite at $31.42 \%$, concrete at $21.23 \%$ and the $\%$ heaviness of the $\mathrm{PVA}^{-} \mathrm{WO}_{3}$ composites varied from 10.6-17.9\%. The results of density and \% heaviness of the composites with respect to lead are presented in Table 4 and Figure 9.

\section{Conclusions}

Polymer composites reinforced with $\mathrm{WO}_{3}$ fillers were successfully fabricated by open mould cast technique. The XRD patterns have revealed an increase in the intensity of $\mathrm{WO}_{3}$ peaks with an increase in filler concentration. The structural studies have revealed the dispersion of filler into a polymer matrix with an average particle size of 5-29 nm. TGA showed a major decrease in weight loss around 340 and $450^{\circ} \mathrm{C}$ with an increase in filler concentration which may be ascribed to the enhanced thermal stability of composites. The study on AC conductivity and UV-vis spectroscopy revealed high resistivity and low conductivity property of $\mathrm{WO}_{3}$ filled PVA composites. The results of $\mathrm{X}$ - and $\gamma$-ray attenuation properties of PVA- $\mathrm{WO}_{3}$ composites at different photon energies showed an increase in $\mu / \rho$ values with filler wt $\%$. The experimental $\mu / \rho$ values were compared with the values obtained from WinXCom. The other attenuation parameters such as HVL, TVL, and $\lambda$ were evaluated to estimate shielding effectiveness. The $\mathrm{Z}_{\text {eff }}$ of composites determined experimentally and theoretically are in good agreement confined to the limitations of Auto- $Z_{\text {eff }}$ software. The density and $\%$ heaviness of composites has confirmed that the fabricated composites are of less weight in comparison with conventional shielding materials. The present studies show that $\mathrm{PVA}-\mathrm{WO}_{3}$ composites may be useful as lightweight radiation shielding materials.
Table 2. Effective atomic number of $\mathrm{WO}_{3}$ filled PVA composites for 5.895 and $6.490 \mathrm{keV}$

\begin{tabular}{cccccc}
\hline \hline \multirow{2}{*}{$\begin{array}{c}\text { Filler wt } \\
\text { \% }\end{array}$} & \multicolumn{2}{c}{$\mathbf{Z}_{\text {eff }}$ at 5.895 keV } & & \multicolumn{2}{c}{$\mathbf{Z}_{\text {eff }}$ at 6.490 keV } \\
\cline { 2 - 3 } \cline { 5 - 6 } WinXCom & Experimental & & WinXCom & Experimental \\
\hline PVA & 11.059 & 11.358 & & 10.945 & 11.290 \\
$5 \%$ & 3.574 & 3.536 & & 3.574 & 3.562 \\
$10 \%$ & 5.442 & 5.441 & & 5.477 & 5.518 \\
$20 \%$ & 9.726 & 9.753 & & 9.885 & 9.944 \\
$30 \%$ & 14.978 & 14.718 & & 15.285 & 14.957 \\
$40 \%$ & 21.603 & 21.369 & & 22.124 & 21.835 \\
\hline \hline
\end{tabular}

Table 3. Effective atomic number of $\mathrm{WO}_{3}$ filled PVA composite for 59.54 and $662 \mathrm{keV}$

\begin{tabular}{|c|c|c|c|c|c|c|}
\hline \multirow{2}{*}{$\begin{array}{l}\text { Filler } \\
\text { wt \% }\end{array}$} & \multicolumn{3}{|c|}{$Z_{\text {eff }}$ at $59.54 \mathrm{keV}$} & \multicolumn{3}{|c|}{$Z_{\text {eff }}$ at $662 \mathrm{keV}$} \\
\hline & WinXCom & $\begin{array}{c}\text { Auto- } \\
\mathbf{Z}_{\text {eff }}\end{array}$ & Experimental & WinXCom & $\begin{array}{c}\text { Auto- } \\
\mathbf{Z}_{\text {eff }}\end{array}$ & Experimental \\
\hline $20 \%$ & 8.936 & 11.567 & 8.760 & -- & -- & -- \\
\hline $30 \%$ & 13.669 & 13.929 & 13.409 & 4.338 & 4.859 & 4.333 \\
\hline $40 \%$ & 19.553 & 15.935 & 19.553 & 4.950 & 5.601 & 4.955 \\
\hline $50 \%$ & 27.353 & 17.818 & 26.509 & 5.743 & 6.579 & 5.724 \\
\hline
\end{tabular}

Table 4. Density and \% heaviness of $\mathrm{WO}_{3}$ filled PVA composites

\begin{tabular}{cccc}
\hline \hline \multirow{2}{*}{ Sample } & \multicolumn{2}{c}{ Density $\mathbf{g ~ c m}^{-3}$} & \multirow{2}{*}{ \% heaviness } \\
\cline { 2 - 3 } & Theoretical & Experimental & \\
\hline Neat PVA & 1.19 & 1.21 & $10.67 \pm 0.41$ \\
$5 \%$ & 1.24 & 1.25 & $11.02 \pm 0.35$ \\
$10 \%$ & 1.30 & 1.29 & $11.37 \pm 0.15$ \\
$20 \%$ & 1.43 & 1.43 & $12.62 \pm 0.31$ \\
$30 \%$ & 1.59 & 1.58 & $13.89 \pm 0.43$ \\
$40 \%$ & 1.79 & 1.79 & $15.79 \pm 0.38$ \\
$50 \%$ & 2.04 & 2.03 & $17.90 \pm 0.52$ \\
\hline \hline
\end{tabular}

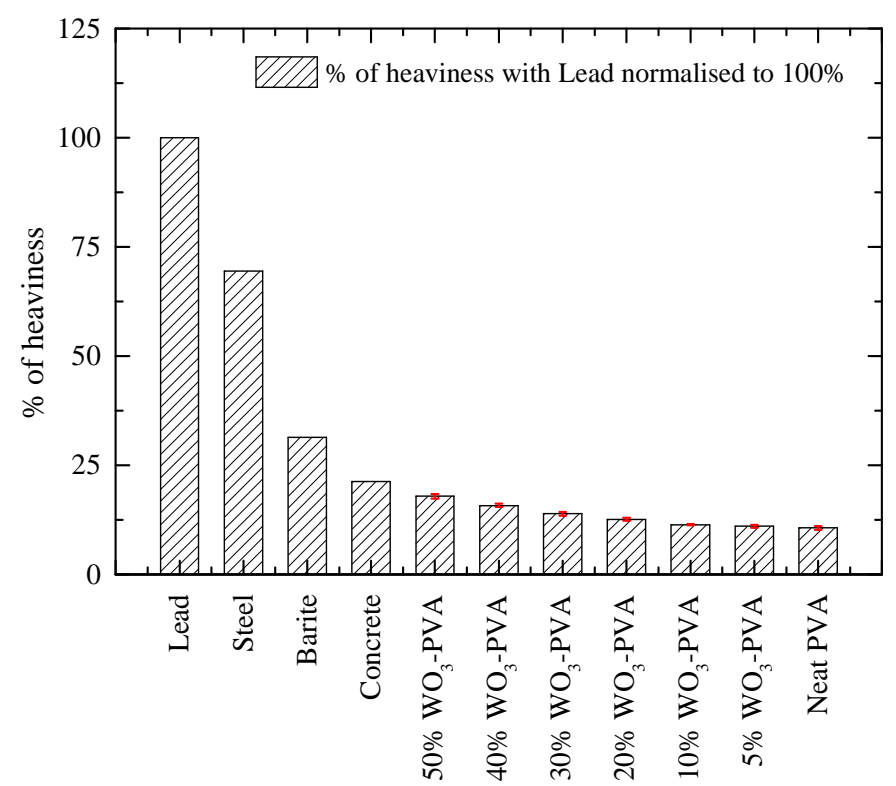

Figure 9. \% heaviness of the polymer composites with respect to traditional shielding materials 


\section{Acknowledgments}

The authors thank the anonymous reviewer for the valued comments and suggestions that greatly improved this manuscript. The X-and $\gamma$-ray shielding study at 5.895, 6.490 and $59.540 \mathrm{keV}$ energies was performed at SSIF, ISRO, Marathalli, Bengaluru. We are extremely thankful to Dr K C Shyama Narendranath and Mr V Koushal of SSIF, ISRO for providing CdTe X-ray detector spectrometer facility, valuable suggestions and discussions during the course of the experiments. Two of the authors (BSG and SBG) acknowledge the financial support from UGC-DAE-CSR, Indore through the project grant CSR-IC/BL-86/CRS-203/2016-19.

\section{References}

[1] Singh N, Singh KJ, Singh K, Singh H. Comparative study of lead borate and bismuth lead borate glass systems as gamma-radiation shielding materials. Nucl Instrum Methods B. 2004;225(3):305-309.

[2] Arbuzov VI, Fyodorov YK. Spectral, radiation-optical and shielding properties of phosphate glasses with high lead content. Adv Mater Res. 2008;39-40:213-218.

[3] Manohara SR, Hanagodimath SM, Gerward L. Photon interaction and energy absorption in glass: A transparent gamma ray shield. J Nucl Mater. 2009;393(3):465-472.

[4] Singh VP, Badiger NM. Shielding efficiency of lead borate and nickel borate glasses for gamma rays and neutrons. Glass Phys Chem. 2015;41(3):276-283.

[5] Kaewkhao J, Limsuwan P. Mass attenuation coefficients and effective atomic numbers in phosphate glass containing $\mathrm{Bi}_{2} \mathrm{O}_{3}, \mathrm{PbO}$ and $\mathrm{BaO}$ at $662 \mathrm{keV}$. Nucl Instrum Methods. 2010;619(1-3):295-297.

[6] Nambiar S, Yeow JTW. Polymer composite materials for radiation protection. ACS Appl Mater Interfaces. 2012;4(11):5717-5726.

[7] Harish V, Nagaiah N, Harish Kumar HG. Lead oxides filled isophthalic resin polymer composites for gamma radiation shielding applications. Indian J Pure Appl Phy. 2012;50(11):847-850.

[8] Li R, Gu Y, Zhang G, Yang Z, Li M, Zhang Z. Radiation shielding property of structural polymer composite: Continuous basalt fiber reinforced epoxy matrix composite containing erbium oxide. Compos Sci Technol. 2017;143:67-74.

[9] Husain HS, Rasheed Naji NA, Mahmood BM. Investigation of gamma ray shielding by polymer composites. IOP Conf Ser: Mater Sci Eng. 2018;454:012131.

[10] Yue K, Luo W, Dong X, et al. A new lead-free radiation shielding material for radiotherapy. Radiat Prot Dosim. 2009;133:256-260.

[11] Wang H, Zhang H, Su Y, et al. Preparation and radiation shielding properties of Gd2O3/PEEK composites. Polym Compos. 2014;36(4):651-659.

[12] Kim Y, Park S, Seo Y. Enhanced X-ray shielding ability of polymer-nonleaded metal composites by multilayer structuring. Ind Eng Chem Res. 2015;54(22):5968-5973.

[13] Soylu HM, Lambrecht Y, Ersöz OA. Gamma radiation shielding efficiency of a new lead-free composite material. J Radioanal Nucl Chem. 2015;305(2):529-534.

[14] Erol A, Pocan I, Yanbay E, et al. Radiation shielding of polymer composite materials with wolfram carbide and boron carbide. Radiat Prot Environ. 2016;39(1):3-6.

[15] Atashi P, Rahmani S, Ahadi B, Rahmati A. Efficient, flexible and lead-free composite based on room temperature vulcanizing silicone rubber/W/Bi2O3 for gamma ray shielding application. J Mater Sci: Mater Electron. 2018;29(14):12306-12322.

[16] AbuAlRoos NJ, Baharul Amin NA, Zainon R. Conventional and new lead-free radiation shielding materials for radiation protection in nuclear medicine: A review. Radiat Phys Chem. 2019;165:108439.

[17] Chai H, Tang X, Ni M, et al. Preparation and properties of novel, flexible, lead-free X-ray-shielding materials containing tungsten and bismuth(III) oxide. J Appl Polym Sci. 2016;133(10):43012.

[18] Ambika MR, Nagaiah N, Harish V, et al. Preparation and characterisation of isophthalic- $\mathrm{Bi}_{2} \mathrm{O}_{3}$ polymer composite gamma radiation shields. Radiat Phys Chem. 2017;130:351-358.

[19] Parvaresh R, Haghparast A, Khoshgard K, et al. An investigation to determine an optimum protective garment material in nuclear medicine. J Biomed Phys Eng. 2018;8(4):381-392.

[20] Gerward L, Guilbert N, Jensen KB, Leving H. WinXCom - a program for calculating X-ray attenuation coefficients. Radiat Phys Chem. 2004;71(3-4):653-654.

[21] Kerur R, Thontadarya SR, Hanumaiah B. Anomalous X-ray attenuation coefficients around the absorption edges using Mn Ka and Cu Ka X-rays. Appl Radiat Isot. 1994;45(2):159-163.

[22] Taylor ML, Smith RL, Dossing F, Franich RD. Robust calculation of effective atomic numbers: The Auto- $Z_{\text {eff }}$ software. Med Phys. 2012;39(4):1769-1778. 
[23] Bhavani S, Pavani Y, Ravi M, et al. Structural and electrical properties of pure and NiCl2 doped PVA polymer electrolytes. Am J Polym Sci. 2013;3(3):56-62.

[24] Chang L, Zhang Y, Liu Y, et al. Preparation and characterization of tungsten/epoxy composites for $\gamma$-rays radiation shielding. Nucl Instrum Methods Phys Res B. 2015;356;88-93.

[25] Rithin Kumar NB, Crasta V, Bhajantri RF, Praveen BM. Microstructural and mechanical studies of PVA doped with ZnO and WO3 composites films. J Polym. 2014; 2014: 1-7.

[26] Hema M, Selvasekerapandian S, Hirankumar G, et alH. Structural and thermal studies of PVA: $\mathrm{NH}_{4} \mathrm{I}$. J Phys Chem Solids. 2009;70(7):1098-1103.

[27] Abd El-Kader KAM, Abdel Hamied SF, Mansour AB, et al. Effect of the molecular weights on the optical and mechanical properties of poly (vinyl alcohol) films. Polym Test. 2002;21(7):847-850.

[28] El-Khodary A. Evolution of the optical, magnetic and morphological properties of PVA films filled with $\mathrm{CuSO}_{4}$. Physica B: Condens Matter. 2010;405(16):3401-3408.

[29] Bhavsar VB, Jha D. Study of refractive index dispersion and optical conductivity of PPy doped PVC films. Indian J Pure App Phys. 2016;54(2):105-110.

[30] Habubi NF, Abed BH, Chiad SS. Optical properties of $\mathrm{BaCl}_{2}$ doped poly(vinyl alcohol) films. Iraqi J Phys. 2012;10(17):18-22. 